\title{
The Relationship Between Blood Lead Level and Preeclampsia
}

\author{
Fatemeh Bayat ${ }^{1}$, Sedigheh Amir Ali Akbari ${ }^{2}$, Atousa Dabirioskoei ${ }^{3}$, Malihe Nasiri ${ }^{4}$, Aliosat Mellati ${ }^{5}$
}

\author{
${ }^{1}$ M.Sc. of Midwifery, Department of Midwifery, Shahid Beheshti University of Medical Sciences, Tehran, Iran \\ ${ }^{2}$ M.Sc. of Midwifery, Department of Midwifery, Faculty of Midwifery and Nursing School, Shahid Beheshti \\ University of Medical Sciences, Tehran, Iran \\ ${ }^{3}$ M.D., Gynecologist, Associate Professor, Department of Gynecology and Obstetrics, Mousavi Hospital, Zanjan \\ University of Medical Sciences, Zanjan, Iran \\ ${ }^{4}$ Ph.D. in Biostatics, Associate Professor, Department of Basic Sciences, Faculty of Shahid Beheshti University of \\ Medical Sciences, Tehran, Iran \\ ${ }^{5}$ Ph.D. in Biochemistry, Professor, Metabolic Research Center and Department of Biochemistry, Faculty of Zanjan \\ University of Medical Sciences, Zanjan, Iran
}

\section{Type of article: Original}

\begin{abstract}
Introduction: Lead is a heavy metal to which people are commonly exposed. One of the possible mechanisms of tissue damages caused by this toxic metal is oxidative stress, which in turn may cause numerous pregnancy complications such as preeclampsia. The present study was conducted to determine the relationship between maternal Blood Lead Level (BLL) and preeclampsia.

Methods: The present case-control study was conducted on 158 pregnant women admitted to a hospital in Zanjan, Iran, from August 2015 to March 2016. To measure their BLL, $1.5 \mathrm{cc}$ of blood was drawn from each participant. The demographic and obstetric details of the patients were recorded in a form. The potentiometric method was used to test the samples. The data obtained were analyzed by SPSS version 22, using Mann-Whitney $\mathrm{U}$ test, the Chi square, independent-samples t-test, Pearson product-moment correlation, and simple linear regression analysis.

Results: The mean BLL was $6.24 \pm 1.74 \mu \mathrm{g} / \mathrm{dl}$ in the control group and $8.04 \pm 3.4 \mu \mathrm{g} / \mathrm{dl}$ in the preeclampsia group. The two groups were matching in terms of the mother's age and education and the household income. A significant relationship was observed between BLL and preeclampsia $(\mathrm{p}=0.028)$, as per every unit of increase $(1$ $\mu \mathrm{g} / \mathrm{dl}$ ) in BLL, systolic blood pressure increased by $0.014 \mathrm{~mm} \mathrm{Hg}$ and diastolic blood pressure by $0.013 \mathrm{~mm} \mathrm{Hg}$ $(\mathrm{p}=0.004)$.

Conclusion: The results obtained suggest a relationship between BLL and preeclampsia. Global health measures should be taken to remove the exposure to lead so as to reduce its absorption by pregnant women.

Keywords: Preeclampsia, Blood lead level (BLL), Pregnancy
\end{abstract}

\section{Introduction}

Preeclampsia and its complications, have turned into one of the main causes of maternal and fetal morbidity and mortality (1), as eclampsia and severe preeclampsia claim 40,000 to 70,000 pregnant women's lives, worldwide every year (2). This complication is more serious in developing countries, where the maternal mortality rate is twenty times as that in developed countries, due to the greater incidence of blood pressure disorders (3). In Iran, preeclampsia is the second leading cause of maternal death, accounting for $18 \%$ of deaths in this group (4). The term gestational hypertension-preeclampsia indicates slight to severe hypertension that involves different organs of the body, ranging from acute gestational hypertension to preeclampsia, eclampsia and the HELLP syndrome (hemolysis,

\section{Corresponding author:}

Sedigheh Amir Ali Akbari, Department of Midwifery, Faculty of Midwifery and Nursing School, Shahid Beheshti University of Medical Sciences, Tehran, Iran.

Tel: +98.9123142669, Fax: +98.2188202512, Email: sedaliakbari@gmail.com

Received: July 23, 2016, Accepted: September 18, 2016, Published: December 2016

iThenticate screening: August 28, 2016, English editing: November 03, 2016, Quality control: December 04, 2016

(C) 2016 The Authors. This is an open access article under the terms of the Creative Commons Attribution-NonCommercialNoDerivs License, which permits use and distribution in any medium, provided the original work is properly cited, the use is non-commercial and no modifications or adaptations are made. 
elevated liver enzymes, low platelet count); (5). Of all the different blood pressure disorders that exist, preeclampsia, whether independently or in combination with chronic hypertension, is considered the most dangerous, and is diagnosed in 3.9\% of all pregnancies (6). This disease is also accountable for complications such as preterm childbirth, perinatal mortality and intrauterine fetal growth retardation (7). Preeclampsia is traditionally defined as a combination of high blood pressure and proteinuria. Modern definitions also include maternal organ dysfunction, such as kidney failure, liver involvement, blood or neurological complications, utero-placental disorder and fetal growth retardation (8). Preeclampsia often occurs after the 20th week of gestation and in the third trimester of pregnancy. If preeclampsia starts before the 32 nd week of gestation, it is called early-onset preeclampsia. This condition also occurs during the postpartum period (9). The cause of preeclampsia remains unknown to date. Nevertheless, inferential data indicate that oxidant-antioxidant imbalance may have a major role in the pathogenesis of the condition (6). One of the main factors involved in oxidative stress is the excessive exposure to toxic metals and the bio-deficiency of the elements required for antioxidant defense mechanisms (10). Lead is one of the heavy metals to which people are commonly exposed (11). The risks associated with lead absorption have been known for centuries. The effects of lead on mental and physical health is an ongoing concern in all countries, including low, medium and indeed high-income countries. No international treaties exist for regulating lead pollution and examining its implications for people's health and lifestyles. The World Health Organization has introduced lead as one of the "ten chemicals of major public health concern" (12). This toxic metal is a natural component of the earth's crust. The excessive use of lead has caused extensive environmental contamination and human exposure as well as substantial public health problems in many different regions of the world. Lead is a toxic and cumulative substance that affects various body systems (13). Lead is a soft grayish-blue heavy metal that enters the body mainly through the respiratory system; it is slightly absorbed by the gastrointestinal system and mostly excreted through feces (14). The exposure to lead causes oxidative stress and the release of reactive oxygen species (ROS) and thus eliminates the essential metals needed for antioxidant reactions from the body. In addition, lead affects the integrity of the cell membranes and their fatty acid composition and causes the release of lipid peroxidases (15). Other mechanisms include a reduction in nitric oxide, an increase in endothelin and thromboxane production and the inhibition of vascular smooth muscle and ATPase activity, thereby leading to an increased blood pressure (16). According to the World Health Organization, in pregnant women, blood lead level (BLL) should be less than $10 \mu \mathrm{g} / \mathrm{dl}$, and according to the guidelines provided by the Center for Disease Control and Prevention, follow-up and interventions should begin immediately in pregnant and lactating women whose BLL exceeds $5 \mu \mathrm{g} / \mathrm{dl}$ (17). The Reabsorption of lead through the bones increases during pregnancy and leads to fetal exposure and poisoning. In female smokers and women with low calcium intake, greater amounts of lead are released from the bones during pregnancy, which is further exacerbated by the nutritional deficiency caused by monotonous diets and little mineral and vitamin intake. The placenta does not appear to protect the fetus from lead, as this metal can reach the fetus through the placenta and accumulate in his body (18). Lead can reach the fetus without difficulty, through passive transportation and can be measurable in the fetal brain before the end of the first trimester $(17,19)$. By the beginning of the 12th week of gestation, lead easily passes through the placenta, reaches the fetus and causes adverse fetal and pregnancy outcomes (20). Previous studies conducted on the relationship between serum BLL and preeclampsia have produced contradictory results. Vigeha et al., found no relationship between BLL and preeclampsia (21), whereas Jameil (22) and Motawi et al., (23) found a significant relationship between the two, Furthermore, the largest lead and zinc mines in the Middle East are located in Zanjan Province and according to the notice of the research committee of Zanjan Provincial General Government, conducting studies on lead is a research priority. The present study was therefore conducted in 2015-2016, in Zanjan, Iran, to examine the relationship between serum BLL and preeclampsia.

\section{Material and Methods}

The present analytical case-control study was conducted from August 2015 to March 2016. This case control study was conducted on pregnant mothers admitted to Ayatollah Mousavi Hospital of Zanjan with a diagnosis of preeclampsia and was approved by the Ethics Committee of Shahid Beheshti University of Medical Sciences. Data were collected through the purposive method. Using the statistical equation below, the minimum sample size required for each group was determined as 78: $\mathrm{n} \geq\left(Z_{\alpha / 2}+Z_{\beta}\right)^{2} \sigma^{2} /\left(\mu_{1}-\mu_{2}\right)$; Where: $\mathrm{n}=$ Sample size; $Z_{\alpha / 2}=1.96$ when $\alpha$ $=0.05 ; Z_{\beta}=0.2$ when $\beta=0.10$ (study power $\left.=80 \%\right) ;\left(\mu_{1}-\mu_{2}\right) / \sigma=0.45$. Iranian women aged 15 to 40 years old, with a singleton pregnancy, at 20 weeks of gestation or more, not suffering from diabetes, lupus or thrombophilia and with no history of alcohol or drug use, with a pre-pregnancy BMI less than $35\left(\mathrm{~kg} / \mathrm{m}^{2}\right)$ and having no family history of preeclampsia and whose pregnancy was not accomplished through IVF were included in the study. The case group (the preeclampsia group) consisted of mothers admitted to the hospital for preeclampsia. High blood pressure, if taken correctly, is diagnosed when systolic pressure exceeds $140 \mathrm{~mm} \mathrm{Hg}$ or diastolic pressure exceeds $90 \mathrm{~mm} \mathrm{Hg}$. 
The American College of Obstetricians and Gynecologists (b2013) proposed new criteria for the diagnosis of preeclampsia in 2013, including proteinuria (a dipstick reading of $1+$ or $2300 \mathrm{mg}$ in 24 hours) or evidence of maternal multi-organ involvement, including thrombocytopenia, renal dysfunction (increased serum creatinine), liver dysfunction (increased liver transaminase), central nervous system disorders (headache, dizziness, blurred vision) or pulmonary edema. The hospital surveyed in this study uses these new criteria for the diagnosis of preeclampsia. Patients developing insulin-dependent diabetes during their course of treatment or being diagnosed with underlying diseases such as the Nephrotic syndrome, ITP and the thrombophilia syndrome were excluded from the study. The control group consisted of healthy eligible mothers with a normal perinatal period admitted to the hospital for the termination of their pregnancy. The researchers followed up with all the patients for preeclampsia until discharge, and excluded them from the study in case they developed preeclampsia. After obtaining informed consent from the participants and completing their demographic, medical and obstetric forms, a $1.5 \mathrm{ml}$ blood sample was taken from each patient and sent at room temperature to the laboratory within less than one hour. The samples were analyzed using the potentiometric method with PSA Ion3 (Steroglass-Italy), which is a highly accurate and sensitive device used for the isolation of heavy metals in both simple and complex environments such as blood, water, foods, chemicals, medications and petroleum and derivatives (23). The reliability of the device was confirmed by testing a single sample ten times, and ensuring that it produced the same results each time. To ensure the uniformity of the tests and the device used, all the tests were carried out in the exact same way and by the same person (a PhD in laboratory sciences). Data were analyzed using SPSS version 22 (IBM@) Corp., Armonk, NY USA). The results obtained were presented as descriptive and inferential statistics. Data normality was checked through Kolmogorov-Smirnov test, and the two groups were compared by using t test for normally distributed quantitative variables, and non-parametric tests for non-normally distributed data. The ANCOVA test was used for adjusting the data. Mann-Whitney U test and Chi square were respectively used for ordinal and qualitative variables. Pearson's $r$ and simple linear regression were used to determine the correlations.

\section{Results}

The mean age of the mothers was $27.37 \pm 6.1$ years old in the control group and $29.67 \pm 6.37$ years old in the preeclampsia group, comprising a significant intergroup difference $(p=0.021)$. The mothers' level of education was significantly lower in the preeclampsia group than in the control group $(\mathrm{p}=0.003)$; (Table1). Similarly, the household income was significantly lower in the preeclampsia group than in the control group $(\mathrm{p}=0.003)$. The infants born in the preeclampsia group were mostly boys $(\mathrm{p}=0.005)$. There were no significant differences between the two groups in terms of the rate of cesarean section; however, when the preeclampsia group was divided into severe and mild categories, a significant difference was observed between the severe preeclampsia and the other two groups, i.e. the mild preeclampsia group and the control group $(\mathrm{p}=0.002)$. Due to the significant difference between the two groups in household income, the mother's level of education and age, data were adjusted using the ANCOVA. A significant relationship was then observed between BLL and preeclampsia $(p=0.028)$. BLL was also significantly correlated with systolic $(\mathrm{r}=0.18$ and $\mathrm{P}=0.02)$ and diastolic $(\mathrm{r}=0.18$ and $\mathrm{p}=0.3)$ blood pressure. This relationship remained significant according to the regression analysis even after adjusting the data pertaining to the mother's age and level of education and household income $(\mathrm{p}=0.004)$, as per each unit of increase in BLL $(1 \mu \mathrm{g} / \mathrm{dl})$, systolic pressure increased by $0.014 \mathrm{mmHg}$ and diastolic pressure by $0.013 \mathrm{mmHg}(\mathrm{p}=0.004)$; (Tables 2$)$.

Table 1. Comparison of the mothers' level of education and household income in the two groups

\begin{tabular}{|l|l|l|l|l|l|}
\hline Variables & Control \% & Preeclampsia \% & p-value \\
\hline \multirow{3}{*}{$\begin{array}{l}\text { Mother's Level of } \\
\text { Education }\end{array}$} & Illiterate & 0 & Illiterate & 6.4 & 0.003 \\
\cline { 2 - 6 } & Below High School Education & 42.3 & Below High School Education & 59 \\
\cline { 2 - 6 } & High School Diploma & 20.5 & High School Diploma & 20.5 \\
\cline { 2 - 6 } & University Degree & 37.2 & University Degree & 15.4 & \\
\hline \multirow{2}{*}{$\begin{array}{l}\text { Household } \\
\text { Income (Rials*) }\end{array}$} & $>10.000 .000$ & 12.8 & $>10.000 .000$ & 35.9 & 0.003 \\
\cline { 2 - 6 } & $10.000 .000-20.000 .000$ & 55.1 & $10.000 .000-20.000 .000$ & 44.9 \\
\cline { 2 - 6 } & $<20.000 .000$ & 33.1 & $<20.000 .000$ & 19.2 & \\
\hline
\end{tabular}

* The Iranian currency

Table 2. Comparison of BLL between the two groups

\begin{tabular}{|l|l|l|l|}
\hline Variables & Control Group & Preeclampsia Group & p-value \\
\hline Systolic Blood Presser $(\mathrm{mmHg})$ & $11.00 \pm 109.52$ & $17.20 \pm 148.33$ & $<0.001$ \\
\hline Diastolic Blood Pressure $(\mathrm{mmHg})$ & $11.3 \pm 68.47$ & $13.14 \pm 95.46$ & $<0.001$ \\
\hline Blood Lead Level $(\mu \mathrm{g} / \mathrm{dl})$ & $1.74 \pm 6.24$ & $3.40 \pm 8.04$ & $<0.001$ \\
\hline
\end{tabular}




\section{Discussion and conclusions}

The present study showed a significant relationship between BLL and preeclampsia. BLL was also found to be significantly correlated with systolic and diastolic blood pressure. In the present study, 32 patients had severe preeclampsia, but no cases of eclampsia were observed. In the follow-up of the control group, no one was found to have preeclampsia or other blood pressure disorders. The Ion 3 device and the potentiometric method were used to analyze the samples. The rate of error was minimized due to the high accuracy of the device (one in a billion). A cross-sectional study by Motawei et al., conducted on 115 preeclampsia patients and 25 healthy pregnant women also showed a significant relationship between lead levels and preeclampsia ( $<<0.001)$; (10). Jameil et al., also examined three groups of women, including the controls, a high-risk preeclampsia group and a preeclampsia group ( $n=40$ per group) and found a significant relationship between the control and preeclampsia groups in lead levels. Lead was also found to be significantly correlated with systolic blood pressure and diastolic blood pressure ( $<0.05)$; (22). In a study by Yazdebek et al., systolic pressure and diastolic pressure were found to be significantly correlated with lead levels after the 24th week of gestation in addition to the positive relationship observed between gestational hypertension and lead levels (11). Ugwuja et al., found a significantly higher lead level in women with hypertension, malaria or low birth weight and concluded that these complications may have been due to the metabolism changes caused by trace elements in the body (19). In a study by Magri et al., conducted on 110 healthy pregnant women and 33 women with gestational hypertension, a significant relationship was observed between lead levels and gestational hypertension. It should be noted that there were no cases of preeclampsia in any of the discussed studies and they yielded a significant relationship between lead levels and gestational hypertension (15). Vigeha et al., found no significant relationships between the mother's lead levels and preeclampsia; in their study, the mean lead level was $5.09 \pm 2.01 \mu \mathrm{g} / \mathrm{dl}$ in the preeclampsia group and $4.82 \pm 2.22 \mu \mathrm{g} / \mathrm{dl}$ in the control group and the mothers were examined after childbirth; however, the present study examined two groups of women before childbirth (21). In a study by Robillard et al., conducted on 3,851 pregnant women, a significant relationship was observed between the mother's systolic/diastolic blood pressure and the concentration of lead in the umbilical cord; however, no relationships were observed between umbilical cord lead and preeclampsia (24). In a cohort study, Rothenberg et al., found no significant relationships between lead levels and blood pressure after adjusting the data (25). The difference between the present study and the discussed studies may be due to the differences in the lead measurement methods used. In the latter two studies, bone and umbilical cord lead levels were measured, while in the present study, lead levels were measured in the mother's blood during the third trimester. According to previous and present findings, there is a distinct relationship between BLL and preeclampsia, systolic blood pressure and diastolic blood pressure. As a toxic metal, lead causes health problems in both developed and developing countries. Measures should therefore be taken to deal with this pervasive problem. The basic measures proposed include the management of serum BLL and assurance that they do not exceed $5 \mu \mathrm{g} / \mathrm{dl}$ in pregnant women, which requires the elimination of the source of lead and prevention of the exposure to lead, the use of lead-containing substances and the pursuit of lead-related activities. An extensive search is required for the numerous sources of lead absorption beyond occupational and environmental ones. Irrespective of the substances consumed, women with pagophagia can benefit from nutritional counseling. Pregnant and lactating women that have or have had serum BLL $\geqslant 5 \mu \mathrm{g} / \mathrm{dl}$ should be assessed for nutritional adequacy and should receive vitamins and nutritional advices with an emphasis on calcium and iron intake. Through raising people's awareness and performing environmental and nutritional interventions, fetal and neonatal exposure to lead can be prevented (13). The present study identified patients with preeclampsia by referring to the definition provided by the American College of Obstetricians and Gynecologists special unit. The points of strength in this study include the follow-up of the control group for the development of preeclampsia until 24-48 hours after childbirth and the measurement of BLL through the potentiometric method and using a PSA Ion3 device (with 1 ppb precision), which has likely minimized the risk of errors in the test results.

\section{Acknowledgments:}

This article was extracted from a research thesis by Fatemeh Bayat for MSc degree entitled "the relationship between BLL and preeclampsia" approved by Shahid Beheshti University of Medical Sciences and supervised by Sedigheh Amir Ali Akbari. The authors would like to express their sincere gratitude to all the women who participated in the study.

\section{Conflict of Interest:}

There is no conflict of interest to be declared.

Authors' contributions:

All authors contributed to this project and article equally. All authors read and approved the final manuscript. 


\section{References:}

1) Feng Y, Xu J, Zhou Q, Wang R, Liu N, Wu Y, et al. Alpha-1 Antitrypsin Prevents the Development of Preeclampsia Through Suppression of Oxidative Stress. Front Physiol. 2016; 7: 176. doi: 10.3389/fphys.2016.00176. PMID: 27303303, PMCID: PMC4882542.

2) Marshal, Raynor. Myles Textbook for Midwives. Churchill Livingstone. 2014; 16th Edition.

3) Sanchez-Aranguren LC, Prada CE, Riano-Medina CE, Lopez M. Endothelial dysfunction and preeclampsia: role of oxidative stress. Front Physiol. 2014; 5: 372. doi: 10.3389/fphys.2014.00372. PMID: 25346691, PMCID: PMC4193194.

4) The Ministry of Health, Treatment and Medical Education. The Mothers' health office annual report, Tehran. 2006.

5) Sibai BM, Stella CL. Diagnosis and management of atypical preeclampsia-eclampsia. Am J Obstet Gynecol. 2009; 200(5): 481.e1-7. doi: 10.1016/j.ajog.2008.07.048. PMID: 19019323.

6) Cunningham FG, Leveno KJ, Bloom SL, Spong CY, Dash JS, Hoffman BL, et al. Williams Obstetrics. 24th edition. New York: McGraw-Hill companies. 2014; 728-80.

7) Uzan J, Carbonnel M, Piconne O, Asmar R, Ayoubi JM. Pre-eclampsia: pathophysiology, diagnosis, and management. Vasc Health Risk Manag. 2011; 7: 467-74. doi: 10.2147/VHRM.S20181. PMID: 21822394, PMCID: PMC3148420.

8) Mol BW, Roberts CT, Thangaratinam S, Magee LA, de Groot CJ, Hofmeyr GJ. Pre-eclampsia. Lancet. 2016; 387(10022): 999-1011. doi: 10.1016/S0140-6736(15)00070-7. PMID: 26342729.

9) American College of Obstetricians and Gynecologists and FAQ. Preeclampsia and high blood pressure during pregnancy. 2014. Available from: http://www.acog.org/Patients/FAQs/Preeclampsia-and-HighBlood-Pressure-During-Pregnancy.

10) Motawei SM, Attalla SM, Gouda HE, El-Harouny MA, El-Mansoury AM. Lead level in pregnant women suffering from pre-eclampsia in Dakahlia, Egypt. Int J Occup Environ Med. 2013; 4(1): 36-44. PMID: 23279796.

11) Yazbeck C, Thiebaugeorges O, Moreau T, Goua V, Debotte G, Sahuquillo J, et al. Maternal blood lead levels and the risk of pregnancy-induced hypertension: the EDEN cohort study. Environ Health Perspect. 2009; 117(10): 1526-30. doi: 10.1289/ehp.0800488. PMID: 20019901, PMCID: PMC2790505.

12) Lu L, Cheng H, Liu X, Xie J, Li Q, Zhou T. Assessment of Regional Human Health Risks from Lead Contamination in Yunnan Province, Southwestern China. PLoS One. 2015; 10(4): e0119562. doi: 10.1371/journal.pone.0119562. PMID: 25893826, PMCID: PMC4404351.

13) World Health Organization. Lead poisoning and health (Fact sheet N 379). Accessed Sept 2013.

14) Dehghani R. Environmental Toxicity. Kashan. Tak Derakht publisher. 2010; 140-43.

15) Lopes AC, Peixe TS, Mesas AE, Paoliello MM. Lead Exposure and Oxidative Stress A Systematic Review. Rev Environ Contam Toxicol. 2016; 236: 193-238. doi: 10.1007/978-3-319-20013-2-3. PMID: 26423075.

16) Magri J, Sammut M, Savona-Venturab C. Lead and other metals in gestational hypertension. Int J Gynaecol Obstet. 2003; 83(1): 29-36. doi: 10.1016/S0020-7292(03)00212-1. PMID: 14511869.

17) Ettinger AS. Guidelines for the identification and management of lead exposure in pregnant and lactating women. US Department of Health and Human Services, Centers for Disease Control and Prevention, National Center for Environmental Health/Agency for Toxic Substances and Disease Registry. 2010.

18) Kim YM, Chung JY, An HS, Park SY, Kim BG, Bae JW, et al. Bio-monitoring of Lead, Cadmium, Total Mercury, and Methylmercury Levels in Maternal Blood and in Umbilical Cord Blood at Birth in South Korea. Int J Environ Res Public Health. 2015; 12(10): 13482-93. doi: 10.3390/ijerph121013482. PMID: 26516876, PMCID: PMC4627044.

19) Ugwuja EI, Ejikeme B, Obuna JA. Impacts of Elevated Prenatal Blood Lead on Trace Element Status and Pregnancy Outcomes in Occupationally Non-exposed Women. Int J Occup Environ Med. 2011; 2(3): 14356. PMID: 23022831.

20) Mokhlesi S, Moghadam Banaem L, Lamieyan M, Aliyan Moghadam N, Safari K. Prediction of preeclampsia based on blood lead levels in pregnant women in the first half of pregnancy. J Shahrekord Univ Med Sci. 2013; 15(6): 44-53.

21) Vigeh M, Yokoyama K, Mazaheri M, Beheshti S, Ghazizadeh S, Sakai T, et al. Relationship between Increased Blood Lead and Pregnancy Hypertension in Women without Occupational Lead Exposure in Tehran, Iran. Arch Environ Health. 2004; 59(2): 70-5. doi: 10.3200/AEOH.59.2.70-75. PMID: 16075900.

22) Jameil NA. Maternal serum lead levels and risk of preeclampsia in pregnant women: a cohort study in a maternity hospital, Riyadh, Saudi Arabia. Int J Clin Exp Pathol. 2014; 7(6): 3182-9. PMID: 25031738, PMCID: PMC4097270. 
23) Brochure of Ion3. Potentiometric Stripping Analysis. Steroglass Italy. 2002: 1-53.

24) Rabinowitz M, Bellinger D, Leviton A, Needleman H, Schoenbaum S. Pregnancy hypertension, blood pressure during labor, and blood lead levels. Hypertension. 1987; 10(4): 447-51. doi: 10.1161/01.HYP.10.4.447. PMID: 3653974.

25) Rothenberg SJ, Kondrashov V, Manalo M, Jiang J, Cuellar R, Garcia M, et al. Increases in Hypertension and Blood Pressure during Pregnancy with Increased Bone Lead Levels. Am J Epidemiol. 2002; 156(12): 1079-87. doi: 10.1093/aje/kwf163. PMID: 12480651. 